\title{
HOMOTOPY MINIMAL PERIOD SELF-MAPS ON FLAT MANIFOLDS WITH CYCLIC HOLONOMIES*
}

\author{
ZHIBIN LIANG ${ }^{\dagger}$ AND XUEZHI ZHAO $\ddagger$
}

\begin{abstract}
This paper studies the homotopical minimal period of self-maps on flat manifolds with cyclic holonomies. We give some necessary conditions on the self-maps on flat manifolds to guarantee that their homotopical minimal periods are infinite. Furthermore, a kind of density of homotopical minimal periods in the natural number set is considered.
\end{abstract}

Key words. Homotopical minimal period, flat manifold, self-map, period point, holonomy.

AMS subject classifications. Primary 37C25; Secondary 55M20.

1. Introduction. Let $X$ be a flat manifold of finite dimension, $f$ a self-map on $X$. The homotopical minimal period of $f$ is defined to be

$$
\operatorname{Hper}(f):=\bigcap_{g \simeq f}\left\{n \in \mathbb{N} \mid \operatorname{Fix}\left(g^{n}\right)-\cup_{k<n} \operatorname{Fix}\left(g^{k}\right) \neq \emptyset\right\}
$$

where $g: X \rightarrow X$ runs over all self-maps homotopic to $f$. The homotopy invariant $\operatorname{Hper}(f)$ reflects very rigid part of the self-map $f$ in dynamical system theory. L. Block, J. Guckenheimer, M. Misiurewicz, L. Young in [2] firstly studied $\operatorname{Hper}(f)$ for self-maps on circles. L. Alsedà, S. Baldwin, J. Llibre, R. Swanson and W. Szlenk in [1] listed completely the homotopical minimal period sets for all self-maps on $T^{2}$, and described some properties of the homotopical minimal period sets for self-maps on tori of arbitrary dimensions. A systematic introduction to this topic can be found in the book [7]. It is suggested by B. Jiang and J. Llibre in [6] that a fundamental question is to determine if the homotopical minimal period of a given self-map is empty, finite or infinite. R. Tauraso in [11] showed that homotopical minimal period of expanding maps on flat manifolds must be co-finite, that is its complement in the set of natural numbers is finite. Later, J. B. Lee, K. B. Lee and Zhao $([9,10])$ showed that the homotopical minimal period of any expanding map is still co-finite.

Let $X$ be an $m$-dimensional flat manifold. By definition, $X$ can be considered as an orbit space $\mathbb{R}^{m} / \Gamma$ of the free action of $\Gamma$ on $X$, where $\Gamma$ fits into following exact sequence

$$
1 \rightarrow \mathbb{Z}^{m} \rightarrow \Gamma \rightarrow G \rightarrow 1
$$

in which $G$ is a finite group, and is said to be the holonomy group of the flat manifold $X$. The group $\Gamma$ is actually a subgroup of isometry group $O(m) \rtimes \mathbb{R}^{m}$ of $\mathbb{R}^{m}$, lying in a bigger group $M(m, \mathbb{Z}) \rtimes \mathbb{R}^{m}$. Thus, the action an element $(B, \beta) \in \Gamma$ on $\mathbb{R}^{m}$ is given by

$$
(B, \beta) \nu=B \nu+\beta \text {. }
$$

\footnotetext{
*Received June 30, 2012; accepted for publication July 16, 2012.

${ }^{\dagger}$ Department of Mathematics, Capital Normal University, Beijing 100048, China (liangzhb@ gmail.com). The first author is supported by National Natural Science Foundation of China (Grant No. 11001183 and 11171231).

$\ddagger$ Department of Mathematics \& Institute of Mathematics and Interdisciplinary Science, Capital Normal University, Beijing 100048, China (zhaoxve@mail.cnu.edu.cn). The second author is supported by National Natural Science Foundation of China (Grant No. 10931005), and a project of Beijing Municipal Education Commission (Grant No. PHR201106118).
} 
Clearly, we have that $\Gamma$ is a semi-product $\mathbb{Z}^{m} \rtimes G$. Since $\Gamma$ acts freely on $\mathbb{R}^{m}$, the space $\mathbb{R}^{m}$ is the universal covering space of the flat manifold $X$.

By [8, Corollary 1.2] (cf. [5, Lemma 2.4]), any self-map $f$ on $X$ is homotopic to a map whose lifting on the universal covering space $\mathbb{R}^{m}$ is an affine map of the form $(U, \mu) \in M(m, \mathbb{Z}) \rtimes \mathbb{R}^{m}$. Such a map is said to be a map induced by $(U, \mu)$ in $M(m, \mathbb{Z}) \rtimes \mathbb{R}^{m}$.

Very recently the authors find some sufficient conditions for the infiniteness of homotopical minimal period of self-map on flat manifold with cyclic holonomy, and obtain more self-maps with infinite homotopical minimal period which are not expanding. The main result is as follows (through out the paper we use $I$ to denote the identity matrix).

Theorem 1.1 (Main Theorem). Let $X$ be an $m$-dimensional flat manifold with holonomy group $G \cong \mathbb{Z} / p \mathbb{Z}$, where $p$ is a prime number with $p \equiv 3 \bmod 4$. Let $f$ be a self-map on $X$ induced by $(U, \mu) \in M(m, \mathbb{Z}) \rtimes \mathbb{R}^{m}$ and $(C, \alpha)$ is a generator of $G$. Assume that

(1) $U C=C^{r} U$ for some integer $r$ with $1 \leqslant r \leqslant p-1$,

(2)

$$
\begin{aligned}
\operatorname{det}\left(I-U^{p-1}\right) \neq 0 & \text { if } r^{2} \neq \equiv 1(\bmod p), \\
\operatorname{det}\left(I-U^{2 p}\right) \neq 0 & \text { if } r^{2} \equiv 1(\bmod p),
\end{aligned}
$$

(3) $x^{p-2} \nmid|x I-U|$.

Then the homotopical minimal period $\operatorname{Hper}\left(f^{2}\right)$ of $f^{2}$ is infinite. Moreover, we have

$$
\liminf _{n \rightarrow \infty} \frac{\#\left\{k \mid k \leq n, k \in \operatorname{Hper}\left(f^{2}\right)\right\}}{\frac{n}{\log n}} \geqslant \frac{1}{\varphi(p(p-1) / 2)},
$$

where $\varphi$ is Euler $\varphi$-function.

The last inequality gives an asymptotic estimation of the density of $\operatorname{Hper}\left(f^{2}\right)$ in the set of natural numbers.

Finally we would like to thank Professor Chunlai Zhao for his help on writing this paper.

2. Two lemmas on matrices. In this section we give two lemmas concerning with matrices.

LEMMA 2.1. Let $C$ and $U$ be two square matrices such that $U C=C^{r} U$ for some positive integer $r$. Then for any integers $i, j$ and $k$,

(1) $U^{i} C^{j}=C^{j r^{i}} U^{i}$.

(2) $\left(C U^{i}\right)^{j}=C^{\sum_{k=0}^{j-1} r^{i k}} U^{i j}$.

(3) $\left(C^{s} U^{i}\right)^{j}=C^{\sum_{k=0}^{j-1}(r s)^{i k}} U^{i j}$.

Proof. All can be proved by induction.

Lemma 2.2. Let $C, U \in M(m, \mathbb{Z})$ be two matrices such that $C^{p}=I, C \neq I, U C=$ $C^{r} U$, where $p$ is a prime, $r$ is a positive integer with $r \not \equiv 0,1(\bmod p)$. Assume that

$$
\begin{aligned}
\operatorname{det}\left(I-U^{p-1}\right) \neq 0 & \text { if } r^{2} \neq 1(\bmod p), \\
\operatorname{det}\left(I-U^{2 p}\right) \neq 0 & \text { if } r^{2} \equiv 1(\bmod p) .
\end{aligned}
$$


If $q$ is a prime with $q \not \equiv 1(\bmod p),(q-1,(p-1) / 2)=1$ and $q>\operatorname{Max}\{m+1, p\}$, then

$$
\operatorname{det}\left(I-C U^{2 q}\right) \neq 0 .
$$

If moreover $x^{p-2} \nmid|x I-U|$, then

$$
C U^{2 q} \neq\left(C^{s} U^{2}\right)^{q} \quad \text { for all integer } s .
$$

Proof. First assume that (2.2) is not true. We are going to prove that $m>q-1$, which contradicts to the condition $q>m+1$.

In the case of $r^{2} \equiv 1(\bmod p)$, From Lemma 2.1 and the condition $C^{p}=I$ we get

$$
\left(C U^{2 q}\right)^{p}=C^{\sum_{k=0}^{p-1} r^{2 q k}} U^{2 p q}=C^{\sum_{k=0}^{p-1} 1} U^{2 p q}=C^{p} U^{2 p q}=U^{2 p q} .
$$

So by the assumption $\operatorname{det}\left(I-C U^{2 q}\right)=0$ we have

$\left.\operatorname{det}\left(I-U^{2 p q}\right)=\operatorname{det}\left(I-C U^{2 q}\right)^{p}\right)=\operatorname{det}\left(I-C U^{2 q}\right) \operatorname{det}\left(I+C U^{2 q}+\cdots+\left(C U^{2 q}\right)^{p-1}\right)=0$.

This means that $U$ has an eigenvalue $\lambda$ with $\lambda^{2 p q}=1$. Let $r^{\prime}$ be the smallest positive integer such that $\lambda^{r^{\prime}}=1$. Then $r^{\prime} \mid 2 p q$. Since $\operatorname{det}\left(I-U^{2 p}\right) \neq 0$, we see that $\lambda^{2 p} \neq 1$, and hence $q \mid r^{\prime}$ (otherwise $\left(r^{\prime}, q\right)=1$ and therefore $r^{\prime} \mid 2 p$, contradict to $\lambda^{2 p} \neq 1$ ). Let $f(x):=\operatorname{det}(x I-U)$ be the characteristic polynomial of $U$, then $f(\lambda)=0$. So $m=\operatorname{deg} f(x) \geqslant[\mathbb{Q}(\lambda): \mathbb{Q}]=\varphi\left(r^{\prime}\right)>q-1$, as desired.

In the case of $r^{2} \not \equiv 1(\bmod p)$, we have

$$
\left(C U^{2 q}\right)^{\frac{p-1}{2}}=C^{\sum_{k=0}^{\frac{p-1}{2}-1} r^{2 q k}} U^{q(p-1)}=C^{\frac{r^{(p-1) q}-1}{r^{2 q}-1}} U^{(p-1) q} .
$$

Since $(\mathbb{Z} / p \mathbb{Z})^{\times}$is a cyclic group of order $p-1$ and $q$ is a prime with $q>p>p-1$, we see that $r^{2 q}=\left(r^{2}\right)^{q} \not \equiv 1(\bmod p)$. But $r^{(p-1) q}=\left(r^{q}\right)^{p-1} \equiv 1(\bmod p)$, so $C^{\frac{r^{(p-1) q}-1}{r^{2 q}-1}}=$ $C^{\frac{1-1}{r^{2 q}-1}}=I$, hence $\left(C U^{2 q}\right)^{\frac{p-1}{2}}=U^{(p-1) q}$. Our assumption $\operatorname{det}\left(I-C U^{2 q}\right)=0$ implies that $\operatorname{det}\left(I-U^{(p-1) q}\right)=0$. Similar to the case above, $U$ has an eigenvalue $\lambda$ with $\lambda^{(p-1) q}=1$. Assume that $r^{\prime}$ is the smallest positive integer such that $\lambda^{r^{\prime}}=1$. Then $r^{\prime} \mid(p-1) q$. From $\operatorname{det}\left(I-U^{p-1}\right) \neq 0$ we deduce that $q \mid r^{\prime}$ and $m \geqslant \varphi\left(r^{\prime}\right)>q-1$, as desired.

Now we prove. Assume (2.3) is not true, that is $C U^{2 q}=\left(C^{s} U^{2}\right)^{q}$. By Lemma 2.1 we have

$$
\left(C^{s} U^{2}\right)^{q}=C^{\sum_{k=0}^{q-1}(r s)^{2 k}} U^{2 q} .
$$

Comparing with the assumption $C U^{2 q}=\left(C^{s} U^{2}\right)^{q}$, we get

$$
\left(C^{\sum_{k=0}^{q-1}(r s)^{2 k}-1}-I\right) U^{2 q}=0 .
$$

We claim that

$$
\sum_{k=0}^{q-1}(r s)^{2 k} \equiv 1(\bmod p) .
$$

In fact, from $C \neq I$ and $C^{p}=I$ we know that among the eigenvalues of $C$ there is a $p$-th primitive root of unity $\zeta_{p}$. Since all entries of $C$ are rational numbers, we 
see that all $\sigma\left(\zeta_{p}\right)$ 's $(\sigma \in \operatorname{Gal}(\overline{\mathbb{Q}} / \mathbb{Q}))$ are eigenvalues of $C$. If $(2.5)$ is not true, then $C^{\sum_{k=0}^{q-1}(r s)^{2 k}-1}-I$ has different eigenvalues $\zeta_{p}^{i}-1(i=1,2, \ldots, p-1)$, and its rank is at least $p-1$. On the other hand, the condition $x^{p-2} \nmid|x I-U|$ says that as an eigenvalue of $U, 0$ is of multiplicity less than $p-2$, and the same happens to $U^{2 q}$. So the rank of $U^{2 q}$ is at least 2. This contradicts to (2.4), and (2.5) is valid.

If $(r s)^{2}-1 \equiv 0(\bmod p)$, then

$$
\sum_{k=0}^{q-1}(r s)^{2 k} \equiv q(\bmod p) .
$$

Combining with $(2.5)$, we get $q \equiv 1(\bmod p)$, which contradicts to the original condition $q \not \equiv 1(\bmod p)$.

If $(r s)^{2}-1 \not \equiv 0(\bmod p)$, by $(2.5)$ we get

$$
(r s)^{2 q}-1 \equiv(r s)^{2}-1(\bmod p),
$$

hence $(r s)^{2(q-1)} \equiv 1(\bmod p)$. Assume that $l$ is the smallest positive integer such that $\left((r s)^{2}\right)^{l} \equiv 1(\bmod p)$. Then $l \mid q-1$. Moreover, from $\left((r s)^{2}\right)^{\frac{p-1}{2}} \equiv 1(\bmod p)$ we get $l \mid \frac{p-1}{2}$. Hence $l \mid((q-1),(p-1) / 2)=1$, a contradiction.

3. Proof of Main Theorem. We first prove a part of the Theorem 1.1 as follows.

THEOREM 3.1. Let $X$ be an $m$-dimensional flat manifold with holonomy group $G \cong \mathbb{Z} / p \mathbb{Z}$, where $p$ is a prime number. Let $f$ be a self-map on $X$ induced by $(U, \mu) \in$ $M(m, \mathbb{Z}) \rtimes \mathbb{R}^{m}$ and $(C, \alpha)$ is a generator of $G$. Assume that

(1) $U C=C^{r} U$ for some $1 \leqslant r \leqslant p-1$,

(2)

$$
\begin{aligned}
\operatorname{det}\left(I-U^{p-1}\right) \neq 0 & \text { if } r^{2} \neq 1(\bmod p), \\
\operatorname{det}\left(I-U^{2 p}\right) \neq 0 & \text { if } r^{2} \equiv 1(\bmod p),
\end{aligned}
$$

(3) $x^{p-2} \nmid|x I-U|$.

Then the set

$$
\{q \mid q \text { is prime }, q>m+1, q \not \equiv 1(\bmod p),(q-1,(p-1) / 2)=1\}
$$

is contained in $\operatorname{Hper}\left(f^{2}\right)$.

Proof. Since the self-map $f$ is induced by $(U, \mu)$, its iteration $f^{2}$ is induced by $\left(U^{2}, \mu+U \mu\right)$. From [5, Theorem 3.1], we obtain that

$$
\begin{aligned}
& \operatorname{Hper}\left(f^{2}\right)=\left\{k \mid \text { there is a }(C, \gamma) \in \Gamma \text { such that } \operatorname{det}\left(I-C\left(U^{2}\right)^{q}\right) \neq 0\right. \text {, } \\
& (C, \gamma)\left(U^{2}, \mu+U \mu\right)^{k} \neq\left((B, \beta)\left(U^{2}, \mu+U \mu\right)^{l}\right)^{k / l} \\
& \text { for all } l \mid k, l \neq k,(B, \beta) \in \Gamma\} \text {. }
\end{aligned}
$$

where $\Gamma=\mathbb{Z}^{m} \rtimes \mathbb{Z} / p \mathbb{Z}$ is the fundamental group of the flat manifold $X$.

To prove $q \in \operatorname{Hper}\left(f^{2}\right)$ it suffices to show that

$$
\operatorname{det}\left(I-C U^{2 q}\right) \neq 0 \text { and } C U^{2 q} \neq\left(C^{s} U^{2}\right)^{q} \quad(\forall s \in \mathbb{Z}) .
$$

(the second inequality ensures that $(C, \gamma)\left(U^{2}, \mu+U \mu\right)^{q} \neq\left((B, \beta)\left(U^{2}, \mu+U \mu\right)^{l}\right)^{q / l}$ for all $l \mid q, l \neq q,(B, \beta) \in \Gamma$ since any $B$ is equal to $C^{s}$ for some integer s.) Note 
that all conditions of Lemma 2.2 are satisfied except $C \neq I$. However, if $C=I$, then $(C, \alpha)^{p}=(I, p \alpha)$. Since $(C, \alpha)$ is of order $p$, we have $(C, \alpha)^{p}=(I, \overrightarrow{0})$. Hence $p \alpha=\overrightarrow{0}$ and $\alpha=\overrightarrow{0}$. So $(C, \alpha)=(I, \overrightarrow{0})$ is the identity of $G$, a contradiction. The conclusion of Theorem 3.1 now follows from Lemma 2.2.

To prove the remaining part of Theorem 1.1 (i.e. formula (1.1)) we need the following Dirichlet Prime Theorem (see [4, p. 13-14]).

THEOREM 3.2. For any two positive coprime integers a and $d$, there are infinitely many primes of the form $a+n d$, where $n \geqslant 0$. Furthermore define $\pi_{a, d}(x):=\#\{p \mid p=$ $a+$ nd for some $n \geqslant 0, p \leqslant x$, and $p$ is a prime $\}$, then

$$
\lim _{x \rightarrow \infty} \frac{\pi_{a, d}(x)}{\frac{x}{\log x}}=\frac{1}{\varphi(d)} .
$$

Proof of (1.1). Since $p \equiv 3 \bmod 4,(p-1) / 2$ is an odd integer. It follows that

$\{q \mid q$ is prime, $q>m+1, q \equiv 2(\bmod p(p-1) / 2)\}$

$\subset\{q \mid q$ is prime, $q>m+1, q \not \equiv 1(\bmod p),(q-1,(p-1) / 2)=1\}$.

By Dirichlet Prime Theorem we have that

$\#\{q \mid q$ is prime, $n \geqslant q, q \equiv 2(\bmod p(p-1) / 2)\}=O\left(\frac{n}{\varphi(p(p-1) / 2) \log n}\right)$.

As $m+1$ is independent of $q$, we also have

$\#\{q \mid q$ is prime, $n \geqslant q>m+1, q \equiv 2(\bmod p(p-1) / 2)\}=O\left(\frac{n}{\varphi(p(p-1) / 2) \log n}\right)$.

Therefore

$$
\liminf _{n \rightarrow \infty} \frac{\#\left\{k \mid k \leq n, k \in \operatorname{Hper}\left(f^{2}\right)\right\}}{\frac{n}{\ln n}} \geqslant \frac{1}{\varphi(p(p-1) / 2)} .
$$

Thus the proof of Main Theorem is completed.

4. An example. In this section we give an example to illustrate the method to determine the homotopical minimal periods applying our Main Theorem (Theorem 1.1).

We consider a Bieberbach group (see [3]) of a 3-dimensional flat manifold

$$
\Gamma=\left\{t_{1}, t_{2}, t_{3}, \alpha \mid \alpha^{3}=t_{1}, \alpha t_{2} \alpha^{-1}=t_{3}, \alpha t_{3} \alpha^{-1}=t_{2}^{-1} t_{3}^{-1}, t_{i} t_{j}=t_{j} t_{i}, i \neq j\right\} .
$$

Its generators can be written in terms of matrices as follows:

$$
t_{2}=\left(I,\left(\begin{array}{l}
0 \\
1 \\
2
\end{array}\right)\right), t_{3}=\left(I,\left(\begin{array}{c}
0 \\
-2 \\
-1
\end{array}\right)\right), \alpha=\left(\left(\begin{array}{ccc}
1 & 0 & 0 \\
0 & 0 & -1 \\
0 & 1 & -1
\end{array}\right),\left(\begin{array}{c}
\frac{1}{3} \\
0 \\
0
\end{array}\right)\right) .
$$

Proposition 4.1. ([10, Proposition 3]) Any endomorphism $\phi$ on $\Gamma$ has the form

$$
\phi(\alpha)=\alpha^{r} t_{2}^{u} t_{3}^{v}, \phi\left(t_{2}\right)=\alpha^{r_{2}} t_{2}^{u_{2}} t_{3}^{v_{2}}, \phi\left(t_{3}\right)=\alpha^{r_{3}} t_{2}^{u_{3}} t_{3}^{v_{3}},
$$


and is in one of following three types:

Type (1): $r=3 k_{1}, u=k_{2}, v=k_{3}, r_{2}=r_{3}=u_{2}=v_{2}=u_{3}=v_{3}=0$;

Type (2): $r=3 k_{1}+1, u=k_{2}, v=k_{3}, r_{2}=r_{3}=0, u_{2}=k_{4}, v_{2}=k_{5}, u_{3}=-k_{5}$, $v_{3}=k_{4}-k_{5}$;

Type (3): $r=3 k_{1}-1, u=k_{2}, v=k_{3}, r_{2}=r_{3}=0, u_{2}=k_{4}, v_{2}=k_{5}$, $u_{3}=-k_{4}+k_{5}, v_{3}=-k_{4}$.

The elements in $M(3, \mathbb{Z}) \rtimes \mathbb{R}^{3}$ inducing self-maps with endomorphisms of above three types are:

$$
\left(\left(\begin{array}{ccc}
3 k_{1} & 0 & 0 \\
3 k_{2}-6 k_{3} & 0 & 0 \\
6 k_{2}-3 k_{3} & 0 & 0
\end{array}\right),\left(\begin{array}{l}
0 \\
0 \\
0
\end{array}\right)\right),\left(\left(\begin{array}{ccc}
3 k_{1}+1 & 0 & 0 \\
0 & k_{4} & -k_{5} \\
0 & k_{5} & k_{4}-k_{5}
\end{array}\right),\left(\begin{array}{c}
0 \\
k_{3}-k_{2} \\
-k_{2}
\end{array}\right)\right)
$$

and

$$
\left(\left(\begin{array}{ccc}
3 k_{1}-1 & 0 & 0 \\
0 & -k_{4} & k_{4}-k_{5} \\
0 & -k_{5} & k_{4}
\end{array}\right),\left(\begin{array}{c}
0 \\
k_{3} \\
k_{3}-k_{2}
\end{array}\right)\right)
$$

where $k_{j}, j=1,2,3,4,5$, are integers.

Let us consider type (3).

LEMMA 4.2. Let $k_{j} \in \mathbb{Z}, j=1,4,5$ and

$$
C=\left(\begin{array}{ccc}
1 & 0 & 0 \\
0 & 0 & -1 \\
0 & 1 & -1
\end{array}\right), U=\left(\begin{array}{ccc}
3 k_{1}-1 & 0 & 0 \\
0 & -k_{4} & k_{4}-k_{5} \\
0 & -k_{5} & k_{4}
\end{array}\right)
$$

Assume that $k_{1} \neq 0$ and $\left(k_{4}, k_{5}\right) \neq(0, \pm 1),(1,0),(1,1),(-1,0),(-1,-1)$, then $U C=$ $C^{2} U, \operatorname{det}\left(I-U^{6}\right) \neq 0$.

Proof. An elementary calculation shows that

$$
U C=C^{2} U=\left(\begin{array}{ccc}
3 k_{1}-1 & 0 & 0 \\
0 & k_{4}-k_{5} & k_{5} \\
0 & k_{4} & k_{5}-k_{4}
\end{array}\right)
$$

To prove $\operatorname{det}\left(I-U^{6}\right) \neq 0$ it suffices to show that all eigenvalues of $U$ are different from 6 -th root of unity. The three eigenvalues of $U$ are $3 k_{1}-1, \pm \sqrt{k_{4}^{2}-k_{4} k_{5}+k_{5}^{2}}$.

If $\left(3 k_{1}-1\right)^{6}=1$, then $3 k_{1}-1= \pm 1$, and $k_{1}=0$.

If $\left( \pm \sqrt{k_{4}^{2}-k_{4} k_{5}+k_{5}^{2}}\right)^{6}=1$, then $k_{4}^{2}-k_{4} k_{5}+k_{5}^{2}=1$. The discriminant of $k_{4}^{2}-k_{4} k_{5}+k_{5}^{2}-1$ in variable $k_{5}$ is $-3 k_{4}^{2}+4$, and it is a square if and only if $k_{4}=0$ or \pm 1 . If $k_{4}=0$, we have that $k_{5}= \pm 1$. If $k_{4}=1$, we have that $k_{5}=0,1$. If $k_{4}=-1$, we have that $k_{5}=0,-1$.

Therefore if $k_{1} \neq 0$ and $\left(k_{4}, k_{5}\right) \neq(0, \pm 1),(1,0),(1,1),(-1,0),(-1,-1)$, we have $\operatorname{det}\left(I-U^{6}\right) \neq 0$.

Proposition 4.3. Let $U$ be the matrix given in Lemma 4.2 with $k_{1} \neq 0,\left(k_{4}, k_{5}\right) \neq$ $(0, \pm 1),( \pm 1,0),(1,1),(-1,-1)$, and $f_{U}$ be the homomorphism induced by $U$. then

$$
\{q \mid q \text { is a prime, } q \equiv 2(\bmod 3) \text { and } q>4\} \subset \operatorname{Hper}\left(f_{U}^{2}\right) \text {. }
$$

Proof. Use the notations in Theorem 3.1. Then $m=3, p=3$. From Lemma 4.2 we have $r=2$ and $\operatorname{det}\left(I-U^{2 p}\right)=\operatorname{det}\left(I-U^{6}\right) \neq 0$. Obviously $x^{p-2}=x \nmid|x I-U|$. So all conditions of Main Theorem are satisfied. By Theorem 3.1 we get the conclusion of Proposition 4.3. 


\section{REFERENCES}

[1] L. Alsedì, S. Baldwin, J. Llibre, R. Swanson, And W. Szlenk, Minimal sets of periods for torus maps via Nielsen numbers, Pacific J. Math., 169 (1995), pp. 1-32.

[2] L. Block, J. Guckenheimer, M. Misiurewicz, and L. Young, Periodic points and topological entropy of one-dimensional maps, in "Global theory of dynamical systems", pp. 18-34, Lecture Notes in Math., 819, Springer, Berlin, 1980.

[3] L. CharlaP, Bieberbach groups and flat manifolds, Universitext, Springer-Verlag, New York, 1986.

[4] G. H. Hardy and E. M. Wright, An Introduction to the Theory of Numbers, 5-th ed. Oxford, England: Clarendon Press, 1979.

[5] Z. Liang AND X. ZhaO, Self-maps on flat manifolds with infinitely many periods, Discrete Contin. Dyn. Syst., 32:6 (2012), pp. 2223-2232.

[6] B. Jiang And J. Llibre, Minimal sets of periods for torus maps, Discrete Contin. Dyn. Syst., 4 (1998), pp. 301-320.

[7] J. Jezierski and W. Marzantowicz, Homotopy methods in topological fixed and periodic points theory, Topological Fixed Point Theory and Its Applications, 3. Springer, Dordrecht, 2006.

[8] K. B. LEE, Maps on infra-nilmanifolds, Pacific J. Math., 168 (1995), pp. 157-166.

[9] J. B. LeE AND K. B. LeE, Lefschetz numbers for continuous maps, and periods for expanding maps on infra-nilmanifolds, J. Geom. Phys., 56 (2006), pp. 2011-2023.

[10] J. B. Lee AND X. ZhaO, Homotopy minimal periods for expanding maps on infra-nilmanifolds, J. Math. Soc. Japan, 59:1 (2007), pp. 179-184

[11] R. TAuraso, Sets of periods for expanding maps on flat manifolds, Monatshefte für Mathematik, 128 (1999), pp. 151-157. 
